\title{
Simulation d'un problème de contact par des méthodes de réduction de modèle pour une application temps réel
}

\author{
Frédéric Druesnea ${ }^{a}$, Bastien Lefèvre, Jean-Luc Dulong et Pierre Villon \\ Dpt GSM, Laboratoire Roberval, Université de Technologie de Compiègne, BP 20529, 60205 Compiègne Cedex, France
}

Reçu le 8 juillet 2010, accepté le 9 juillet 2010

\begin{abstract}
Résumé - Les outils de CAO et de réalité virtuelle sont utiles pour simuler en temps réel la manipulation de pièces rigides, avec une détection des collisions. Les simulateurs temps réel industriels ont également besoin de prendre en compte les pièces déformables; cela suppose de pouvoir calculer en temps réel la déformée de pièces en contact. La méthode des éléments-finis ne peut pas fournir la déformée en temps réel pour un modèle à non-linéarités géométrique, matérielle et de contact. Une méthodologie composée de deux phases est ici proposée. Tout d'abord, une phase d'apprentissage consiste à réaliser une campagne de précalculs par éléments-finis représentative des besoins de la simulation en temps réel. Puis, la phase temps réel, non exposée dans cet article, exploite par interpolation la surface de réponse obtenue lors de cette campagne de précalculs. L'objectif de cet article, dans le cadre de la phase d'apprentissage, est de construire une surface de réponse réduite; pour cela des méthodes de réduction de modèle sont utilisées. Trois niveaux de réduction sont étudiés : une méthode adaptative avec base, la technique POD, et l'hyper réduction. L'écrasement d'un cylindre en caoutchouc sera étudié afin d'évaluer la performance de ces différentes méthodes.
\end{abstract}

Mots clés : Méthode adaptative / hyper réduction / POD / surface de réponse réduite / contact mécanique

\begin{abstract}
Simulation of a contact problem using model reduction techniques. CAD and virtual reality tools are useful to simulate the manipulation of rigid parts, and particularly their collisions. Real time simulation need to take into account the deformation of flexible part; this suppose the computation of the deformation of parts in real time, with mechanical contact. The finite-element method can not allow a real-time response for a non-linear mechanical model (geometry, material, mechanical contact). A methodology divided into two phases is proposed. First, the learning phase consists on a calculation campaign of characteristic load cases, in respect of the requirements of the real time simulation. Then, the real time phase, used the results of the first phase by interpolation. Only the first one is exposed in this paper. The aim is to obtain quickly a reduced response. In order to attempt this goal, model reduction methods are used. We focus on three reduction levels : adaptive method, POD approach, and hyper reduction. A crush of a cylinder in rubber is used to expose the performance of the model reduction methods.
\end{abstract}

Key words: Adaptive method / hyper reduction / POD / reduced response surface / mechanical contact

\section{Introduction}

Le recours à la simulation numérique dans le processus de conception de systèmes mécaniques est fréquent. Les enjeux sont de réduire les délais, d'assurer la fiabilité de la conception, et d'optimiser. Les constructeurs de systèmes de transport (aéronautique, automobile, ... ) ont été précurseurs dans l'utilisation des outils de simu-

\footnotetext{
${ }^{a}$ Auteur pour correspondance : fdruesne@utc.fr
}

lation. Ces grandes entreprises le sont maintenant dans le développement et l'utilisation de simulateurs temps réel car les outils de réalité virtuelle sont une aide à la validation d'un prototype. Des simulateurs à retour d'effort [1] sont aujourd'hui opérationnels dans ces industries, pour la manipulation de pièces rigides. La difficulté reste de simuler en temps réel la déformation de pièces souples.

De nombreux modèles déformables ont été développés en infographie pour des besoins d'animations vidéo, souvent à base de modèles physiques simplifiés. Toutefois, 


\section{Nomenclature}

\begin{tabular}{|ll|}
\hline $\boldsymbol{K}_{\mathrm{T}}$ & matrice de rigidité tangente \\
$\boldsymbol{r}$ & résidu numérique \\
$\boldsymbol{u}$ & déplacement \\
$n$ & nombre de degrés de liberté \\
$\boldsymbol{\Phi}$ & base modale \\
$\boldsymbol{a}$ & vecteur de coefficients \\
$m$ & nombre de modes \\
$\hat{\boldsymbol{K}}_{\mathrm{T}}$ & matrice de rigidité tangente réduite \\
$\hat{\boldsymbol{r}}$ & résidu réduit \\
$m_{\text {limit }}$ & valeur limite de nombre de modes \\
$m$ & nombre réduit de modes avec la POD \\
$\boldsymbol{P}$ & matrice de sélection de degré de liberté pour l'hyper réduction \\
\hline
\end{tabular}

nous pouvons citer James et Pai [2] qui utilisent les modes propres obtenus par la méthode des éléments-finis.

La chirurgie endoscopique est très active dans le développement de simulateur temps réel, notamment pour la formation des chirurgiens ou les opérations à distance. La méthode des éléments-finis est parfois utilisée [3] pour simuler des comportements élastiques.

Le développement de simulateur temps réel pour des modèles déformables, avec non-linéarités, est plus rare. Les travaux de Duriez [4] et de Talbi [5] proposent une simulation temps réel dans un contexte avec contact mécanique et retour d'effort; les calculs nécessaires à la simulation sont réalisés en temps réel. Toutefois, lorsque le modèle est à fortes non-linéarités, le précalcul est incourtournable, Niroomandi [6] propose un modèle déformable temps réel de cornée humaine, avec utilisation de techniques de réduction de modèle. Cette approche est a posteriori, une campagne de calculs est réalisée, puis la technique POD [7] est appliquée afin de constituer une base modale représentative de la campagne, utile à la phase temps réel.

Notre approche est de proposer une méthodologie qui se décompose également en deux phases [8] afin de pouvoir résoudre tout type de problème non-linéaire, sans hypothèse mécanique simplificatrice. Notre phase d'apprentissage a comme objectif de construire une surface de réponse réduite, qui sera ensuite utilisée dans la phase de simulation temps réel proprement dite. Afin de minimiser le volume de données utiles à la phase temps réel et de réduire le temps de précalcul, ces méthodes de réduction sont utilisées pour la construction de la surface de réponse. La réduction de modèle a pour but ici de réduire le nombre de degrés de liberté du système à résoudre. Trois niveaux de réduction de modèle sont ici présentés. Le premier consiste à représenter le champ de déplacement en projection sur une base modale construite de manière adaptative, de dimension largement inférieure au nombre de degrés de liberté du système. Le second niveau de réduction est l'utilisation de la technique POD qui va permettre de calculer des modes optimaux, et en nombre réduit, de cette base modale. Enfin, le troisième niveau de réduction porte sur la résolution dite hyper réduite [9] dans laquelle, seuls quelques degrés de liberté du modèle complet seront utilisés.

Pour ces méthodes, cet article vise à étudier les formulations de Galerkin et de minimisation du résidu. En raison du contexte d'utilisation en temps réel pour la réalité virtuelle, nous nous intéressons à un problème de contact.

L'application choisie, pour mettre au point ces méthodes de réduction de modèle, est l'écrasement d'un cylindre de rayon $15 \mathrm{~mm}$ en caoutchouc sur un solide indéformable. La campagne de calculs correspondante est composée de 70 cas de charges successifs dans la même direction, pour atteindre un écrasement de $20 \%$. Le modèle matériel néo-hookéen est utilisé pour traiter l'hyperélasticité du caoutchouc avec un module de Young de 8,76 MPa. La résolution du contact unilatéral sans frottement est effectuée avec la méthode du lagrangien augmenté [10]. Le modèle choisi est 2D en déformation plane avec une symétrie, le maillage est constitué d'élémentsfinis Q4 à 3200 degrés de liberté. Cette application comporte donc les non-linéarités géométrique, matérielle et de contact.

\section{Méthode adaptative de réduction de modèle}

La méthode numérique proposée ici, afin de résoudre un problème de contact mécanique entre un solide déformable hyperélastique et un solide rigide, est adaptative.

En effet, le champ de déplacement $\boldsymbol{u}$ inconnu va être projeté sur une base modale, qui va évoluer au cours de la résolution : c'est le premier niveau de réduction. Puis, pour éviter que cette base modale ne devienne trop importante en dimension, une technique POD (Proper Orthogonal Decomposition) est régulièrement appliquée pour ne conserver que les modes les plus significatifs : c'est le second niveau de réduction. Enfin, nous présentons une variante de cette méthode, la méthode d'hyper réduction, pour laquelle nous effectuons la résolution sur un nombre réduit de degrés de liberté : c'est le troisième niveau de réduction. 
La phase d'apprentissage, précédemment citée, consiste en la réalisation d'une campagne de calculs, une succession de cas de charge modélisant l'écrasement du cylindre en caoutchouc.

Le problème à résoudre $\boldsymbol{r}(\boldsymbol{u})=0$ en champ de déplacement $\boldsymbol{u}$ a la forme suivante (1), et comporte $n$ degrés de liberté :

$$
\boldsymbol{K}(\boldsymbol{u}) \boldsymbol{u}-\boldsymbol{f}(\boldsymbol{u})=\boldsymbol{r}(\boldsymbol{u})
$$

Un schéma de type Newton-Raphson, linéarisé par un développement de Taylor au premier ordre, mène à l'écriture itérative (2), en considérant l'incrément de déplacement $\mathrm{d} \boldsymbol{u}=\boldsymbol{u}^{i}-\boldsymbol{u}^{i-1}$ :

$$
\boldsymbol{r}\left(\boldsymbol{u}^{i}\right)=\boldsymbol{r}\left(\boldsymbol{u}^{i-1}\right)+\frac{\partial \boldsymbol{r}}{\partial \boldsymbol{u}}\left(\boldsymbol{u}^{i-1}\right) \mathrm{d} \boldsymbol{u}
$$

À l'itération $i$ de Newton-Raphson, le résidu $\boldsymbol{r}\left(\boldsymbol{u}^{i-1}\right)$ et la matrice de rigidité tangente $\boldsymbol{K}_{\mathrm{T}}\left(\boldsymbol{u}^{i-1}\right)=\frac{\partial \boldsymbol{r}}{\partial \boldsymbol{u}}\left(\boldsymbol{u}^{i-1}\right)$ sont connus dans l'équation (2), le résidu $\boldsymbol{r}\left(\boldsymbol{u}^{i}\right)$ est donc linéaire en $\mathrm{d} \boldsymbol{u}$, et il est noté $\boldsymbol{R}(\mathrm{d} \boldsymbol{u})$. Cette boucle itérative de Newton-Raphson est résolue pour une configuration des solides en contact, et s'inclut donc dans une boucle de contact qui sera décrite par la suite.

Le coût itératif du schéma de Newton-Raphson, consistant à annuler l'équation (2), réside dans la formation et l'inversion de la matrice de rigidité tangente $\boldsymbol{K}_{\mathrm{T}}$ de dimension $n$. Afin de réduire ce coût, une méthode adaptative avec base modale est utilisée pour décrire le champ de déplacement.

\subsection{Méthode adaptative}

Le chargement est représenté par une succession de cas de charge $k$, chaque déplacement $\boldsymbol{u}_{k}$ correspondant est projeté sur une base modale $\boldsymbol{\Phi}=\left\{\phi_{1}, \ldots, \phi_{m}\right\}$ composée de $m$ modes. L'intérêt est ici dans la dimension $m$, très réduite par rapport au nombre $n$ de degrés de liberté. Le déplacement est ainsi représenté par la forme $\boldsymbol{u}=\boldsymbol{\Phi} \boldsymbol{a}$, avec $\boldsymbol{a}$ un vecteur de coefficients. L'équation itérative (2) devient alors la forme suivante (3) en da pour le cas de charge $k$.

$$
\boldsymbol{R}(\mathrm{d} \boldsymbol{a})=\boldsymbol{r}\left(\boldsymbol{a}_{k}^{i-1}\right)+\boldsymbol{K}_{\mathrm{T}}\left(\boldsymbol{a}_{k}^{i-1}\right) \boldsymbol{\Phi} \mathrm{d} \boldsymbol{a}
$$

Ce système est composé de $n$ équations pour $m$ inconnues. Le problème est surdéterminé, nous cherchons alors à minimiser la fonction coût $J_{\mathrm{Q}}(4)$ du résidu $\boldsymbol{R}$ pour une norme $Q$.

$$
J_{\mathrm{Q}}(\mathrm{d} \boldsymbol{a})=\frac{1}{2}\|\boldsymbol{R}\|_{\mathrm{Q}}^{2}=\frac{1}{2} \boldsymbol{R}^{\mathrm{T}}(\mathrm{d} \boldsymbol{a}) \boldsymbol{Q} \boldsymbol{R}(\mathrm{d} \boldsymbol{a})
$$

La dérivée de la fonction coût (4) dans la direction d $\boldsymbol{a}$, pour tous les vecteurs $\boldsymbol{\delta}$ d $\boldsymbol{a}$, mène à l'expression (5).

$$
\left\langle J_{\mathrm{Q}}^{\prime}(\mathrm{d} \boldsymbol{a}), \boldsymbol{\delta} \mathrm{d} \boldsymbol{a}\right\rangle=\boldsymbol{R}^{\mathrm{T}} \boldsymbol{Q} \boldsymbol{K}_{\mathrm{T}} \boldsymbol{\Phi}
$$

Le minimum de la fonction coût (4) est ainsi obtenu pour l'équation itérative suivante (6), dans laquelle nous distinguons la forme $\hat{\boldsymbol{K}}_{\mathrm{T}} \mathrm{d} \boldsymbol{a}=-\hat{\boldsymbol{r}}$.

$$
\left[\boldsymbol{\Phi}^{\mathrm{T}} \boldsymbol{K}_{\mathrm{T}}^{\mathrm{T}} \boldsymbol{Q} \boldsymbol{K}_{\mathrm{T}} \boldsymbol{\Phi}\right] \mathrm{d} \boldsymbol{a}=-\boldsymbol{\Phi}^{\mathrm{T}} \boldsymbol{K}_{\mathrm{T}}^{\mathrm{T}} \boldsymbol{Q} \boldsymbol{r}
$$

Nous allons maintenant discuter du choix de la norme $Q$ [11], et de son incidence sur la matrice de rigidité tangente réduite $\hat{\boldsymbol{K}}_{\mathrm{T}}$ et sur le résidu réduit $\hat{\boldsymbol{r}}$. Deux possibilités sont ici présentées pour la norme $Q$.

Pour une norme $Q=\boldsymbol{K}_{\mathrm{T}}^{-1}$, la fonction coût $J=$ $\frac{1}{2}\|\boldsymbol{R}\|_{\boldsymbol{K}_{\mathrm{T}}^{-1}}^{2}$ mène à l'équation à résoudre (7), correspondant à une formulation de Galerkin.

$$
\left[\boldsymbol{\Phi}^{\mathrm{T}} \boldsymbol{K}_{\mathrm{T}} \boldsymbol{\Phi}\right] \mathrm{d} \boldsymbol{a}=-\boldsymbol{\Phi}^{\mathrm{T}} \boldsymbol{r}
$$

Et pour une norme $Q=\boldsymbol{I}$, la fonction coût $J=\frac{1}{2}\|\boldsymbol{R}\|_{\mathrm{L}_{2}}^{2}$ conduit à une formulation en minimisation du résidu avec la forme suivante (8).

$$
\left[\boldsymbol{\Phi}^{\mathrm{T}} \boldsymbol{K}_{\mathrm{T}}^{\mathrm{T}} \boldsymbol{K}_{\mathrm{T}} \boldsymbol{\Phi}\right] \mathrm{d} \boldsymbol{a}=-\boldsymbol{\Phi}^{T} \boldsymbol{K}_{\mathrm{T}}^{\mathrm{T}} \boldsymbol{r}
$$

Nous allons tester ensuite ces deux choix de formulation sur notre application de cylindre en caoutchouc écrasé sur un plan rigide dans section 3.1.

Pour les équations à résoudre (7) et (8), la matrice de rigidité tangente réduite $\hat{\boldsymbol{K}}_{\mathrm{T}}$ est de dimension $m \times m$, et le vecteur résidu réduit $\hat{\boldsymbol{r}}=\boldsymbol{\Phi}^{\mathrm{T}} \boldsymbol{K}_{\mathrm{T}}^{\mathrm{T}} \boldsymbol{r}$ est de dimension $m$. Cette matrice $\hat{\boldsymbol{K}}_{\mathrm{T}}$ est pleine, toutefois son inversion sera bien moins coûteuse que l'inversion de la matrice bande de rigidité tangente $\boldsymbol{K}_{\mathrm{T}}$ de dimension $n \times n$ de l'équation (3).

Ces équations itératives (7) et (8) sont l'objet de la résolution de la boucle de Newton-Raphson (I) de l'algorithme de la figure 1. Cette boucle est incluse dans une boucle d'enrichissement de la base modale (II).

En effet pour chaque forme itérative (7) et (8), consistant à déterminer le vecteur des coefficients $\boldsymbol{a}$, la base modale $\boldsymbol{\Phi}$ est supposée connue. Cette base est initialisée avec un premier cas de charge calculé à l'aide d'itérations Newton-Raphson sur $\boldsymbol{u}$, ce qui permet d'aboutir au premier mode $\Phi_{1}(9)$ et au premier coefficient $\boldsymbol{a}_{1}(10)$.

$$
\begin{gathered}
\Phi_{1}=\frac{\boldsymbol{u}_{1}}{\left\|\boldsymbol{u}_{1}\right\|} \\
\boldsymbol{a}_{1}=\left\|\boldsymbol{u}_{1}\right\|
\end{gathered}
$$

Une fois l'initialisation de la base modale réalisée, la réalisation de la campagne de calculs va nécessiter l'adaptation de la base en vue d'assurer la convergence pour chaque cas de charge; le modèle est en effet à nonlinéarités géométrique, matérielle et de contact. Lorsque le critère en résidu n'est pas satisfait (Fig. 1), un mode est calculé par une itération de Newton-Raphson $\mathrm{d} \boldsymbol{u}=$ $\left(\boldsymbol{K}_{\mathrm{T}}\right)^{-1} \boldsymbol{r}$, et ce mode est ajouté à la base modale $\boldsymbol{\Phi}$. Nous avons testé plusieurs alternatives pour enrichir la base modale, notamment un enrichissement avec le résidu. Mais il s'est avéré que, pour notre modèle, l'inversion de 
Boucle de contact (III)

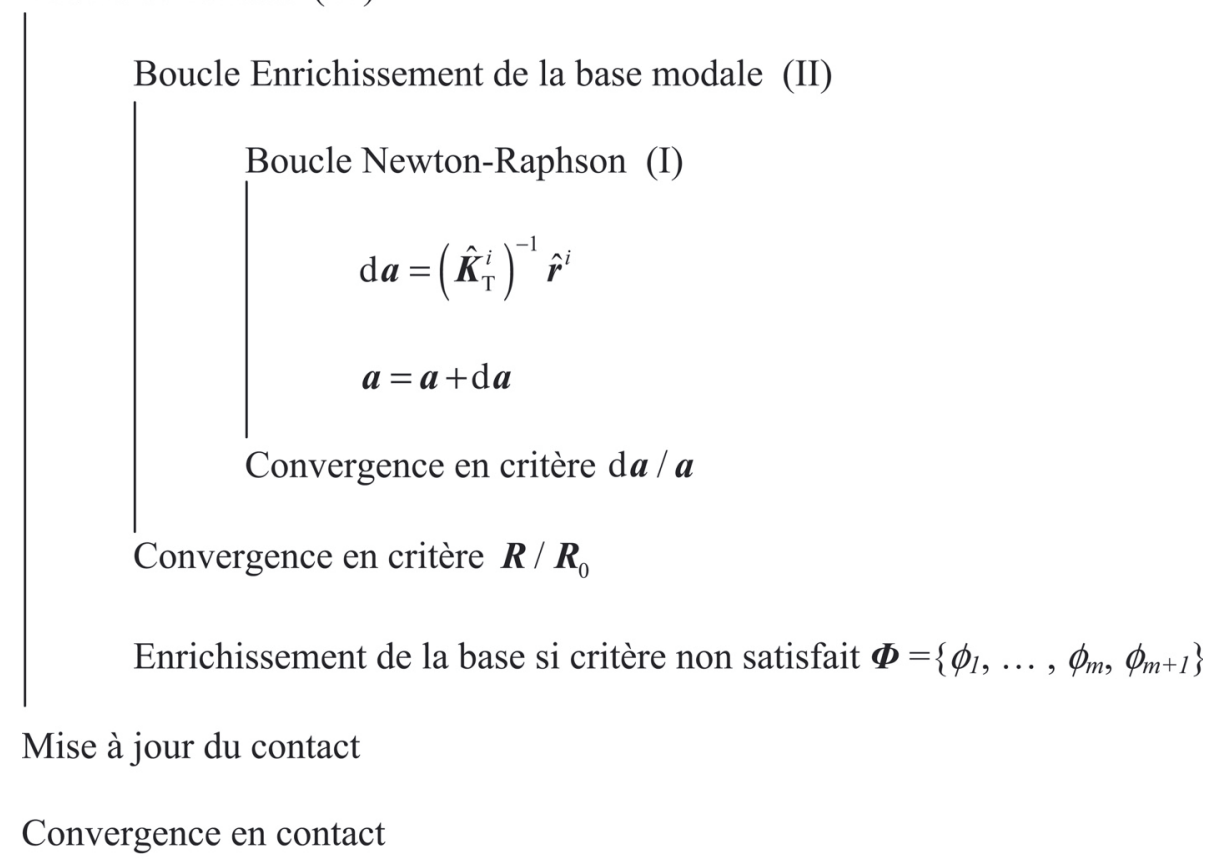

\section{Réduction de la base modale $\boldsymbol{\Phi}^{\prime}=\boldsymbol{\Phi} \psi$ avec la technique POD}

Fig. 1. Algorithme de résolution pour un cas de charge $k$.

la matrice tangente était plus efficace que de multiples enrichissements avec des résidus successifs. Ceci a un coût numérique, toutefois le nombre d'enrichissements est bien inférieur au nombre d'itérations de Newton-Raphson, ce que nous allons observer dans l'application numérique aux sections 3.1 et 3.2 .

Le nouveau mode (11) est orthogonalisé par un schéma de Gram-Schmidt et normalisé (12) dans le but d'obtenir une nouvelle base $\boldsymbol{\Phi}=\left\{\phi_{1}, \ldots, \phi_{m}, \phi_{m+1}\right\}$.

$$
\begin{gathered}
v=\mathrm{d} \boldsymbol{u}-\boldsymbol{\Phi} \boldsymbol{\Phi}^{\mathrm{T}} \mathrm{d} \boldsymbol{u} \\
\Phi_{m+1}=\frac{v}{\|v\|} .
\end{gathered}
$$

L'algorithme de cette résolution adaptative est donc caractérisé par une double boucle (Fig. 1), remplaçant la simple boucle Newton-Raphson en déplacement $\boldsymbol{u}$. La boucle itérative Newton-Raphson porte ici sur la résolution de l'équation (7) ou (8) sur les coefficients $\boldsymbol{a}$, incluse dans une boucle d'enrichissement de la base modale $\boldsymbol{\Phi}$.

Nous allons maintenant présenter la décomposition orthogonale aux valeurs propres, qui va nous permettre de calculer quelques modes représentatifs de cette base modale $\boldsymbol{\Phi}$, avec comme intérêt de réduire la taille de la base.

\subsection{Décomposition orthogonale aux valeurs propres}

La décomposition orthogonale aux valeurs propres (Proper Orthogonal Decomposition POD) a été introduite en 1967 par Lumley en mécanique des fluides [12].
Cette technique est la plus fréquente pour la réduction de données. Elle est utile dans divers domaines comme l'analyse de données, le traitement d'image ou la mécanique des fluides avec une approche de la POD par la méthode Snapshot [13], permettant de réduire considérablement la taille du problème à résoudre. Le concept d'une méthode adaptative avec POD est également utilisée dans des travaux récents de mécanique des fluides [14].

La POD consiste à rechercher une base orthogonale qui approxime au mieux le champ.

Nous appliquons cette technique à notre problème de contact mécanique pour un matériau hyperélastique en vue d'obtenir une base modale optimale. Quelques modes représentatifs suffisent à décrire la base modale, ce qui réduit le nombre $m$ d'équations de (7) et (8).

Notre problème, décrit précédemment, est à non linéarités géométrique, matérielle, et de contact. La réalisation de la campagne de calculs nécessite donc d'enrichir la base modale $\boldsymbol{\Phi}$. A l'issue de chaque cas de charge, la POD est donc possiblement appliquée sur les coefficients $\boldsymbol{a}$, si la taille de la base est considérée trop importante $m>m_{\text {limit }}$ (Fig. 1). Au cas de charge $k$ de la campagne de calculs, le déplacement s'écrit alors sous la forme (13).

$$
\left\{\boldsymbol{u}_{1}, \ldots, \boldsymbol{u}_{k}\right\}=\left\{\boldsymbol{\Phi} \boldsymbol{a}_{1}, \ldots, \boldsymbol{\Phi} \boldsymbol{a}_{k}\right\}
$$

La POD nécessite le centrage des données, c'est à dire l'équation (14) décrivant la moyenne des coefficients et le 
centrage (15).

$$
\begin{aligned}
& \overline{\boldsymbol{a}}_{k}=\frac{1}{k} \sum_{j=1}^{k} \boldsymbol{a}_{j} \\
& \hat{\boldsymbol{a}}_{j}=\boldsymbol{a}_{j}-\overline{\boldsymbol{a}}_{k}
\end{aligned}
$$

La matrice de covariance $\boldsymbol{C}_{k}$ des coefficients $\boldsymbol{a}$ est ensuite calculée, au cas de charge $k$, sous la forme suivante (16).

$$
\boldsymbol{C}_{k}=\sum_{j=1}^{k} \hat{\boldsymbol{a}}_{j} \hat{\boldsymbol{a}}_{j}^{\mathrm{T}}
$$

La nouvelle base modale est constituée de $m^{\prime}$ modes, les $m^{\prime}$ premiers vecteurs propres de la matrice de covariance $\boldsymbol{C}_{k}$ correspondant aux valeurs propres $\lambda$. Les $m^{\prime}$ valeurs propres sont sélectionnées avec le critère (17), avec $\lambda_{\max }$ la plus grande valeur propre et $\varepsilon_{\text {red }}$ une limite fixée dans notre cas à $10^{-8}$.

$$
\frac{\lambda}{\lambda_{\max }}>\varepsilon_{\text {red }}
$$

Les $m^{\prime}$ vecteurs propres, correspondant aux $m^{\prime}$ valeurs propres les plus grandes, sont stockés dans une matrice $\psi$. La nouvelle base modale $\boldsymbol{\Phi}^{\prime}$, qui va être utilisée pour décrire le déplacement, est l'actualisation de la base précédente $\boldsymbol{\Phi}$ avec la matrice $\psi$ des vecteurs propres, soit la forme (18), cette base est de dimension réduite $n \times m^{\prime}$.

$$
\boldsymbol{\Phi}^{\prime}=\boldsymbol{\Phi} \psi
$$

Les nouveaux coefficients $\hat{\boldsymbol{a}}^{\prime}$ associés, de dimension $\mathrm{m}^{\prime}$, sont calculés avec l'expression suivante (19).

$$
\hat{\boldsymbol{a}}^{\prime}=\psi^{\mathrm{T}} \hat{\boldsymbol{a}}
$$

L'application de la technique POD, sur le champ déplacement, fournit ainsi un champ approché (20).

$$
\tilde{\boldsymbol{u}}_{j}=\boldsymbol{\Phi}^{\prime} \hat{\boldsymbol{a}}_{j}^{\prime}+\overline{\boldsymbol{u}}
$$

La mise à jour de la base modale, avec la technique POD, a ici un double intérêt. D'une part, cela permet de réduire le nombre de modes, et le coût de la matrice de rigidité tangente réduite (7) et (8). Et d'autre part, les modes calculés sont optimaux.

Cette technique de réduction de données est mise en place dans notre algorithme (Fig. 1) à l'issue de la convergence de la boucle de contact.

\subsection{Méthode adaptative d'hyper réduction}

La méthode adaptative d'hyper réduction, proposée par Ryckelynck $[9,15]$, est une variante de la méthode précédemment présentée à la section 2.1; elle a déjà été appliquée pour résoudre des problèmes de thermique transitoire et d'évolution élasto-plastique. Cette méthode adaptative apporte un nouveau niveau de réduction de modèle en résolvant le système sur quelques degrés de liberté bien choisis. Nous allons l'appliquer à notre problème de contact mécanique pour un matériau hyperélastique.

Cette méthode adaptative d'hyper réduction consiste en effet à construire la matrice de rigidité tangente réduite des équations itératives (7) et (8) uniquement pour certains degrés de liberté caractéristiques. Le coût de la construction de la matrice de rigidité tangente réduite va de nouveau être réduit, ainsi que son inversion.

Nous reprenons la fonction coût $J_{Q}(4)$, et nous allons chercher ici à minimiser le résidu hyper réduit $\boldsymbol{P} \boldsymbol{R}(21)$ au sens de la norme $Q$. La matrice $\boldsymbol{P}$ permet de sélectionner les degrés de liberté que l'on souhaite conserver pour la résolution du système; c'est une matrice identité creuse : le terme vaut 1 lorsque le degré de liberté est sélectionné. Nous verrons, pour l'application du cylindre écrasé, quels sont les degrés de liberté judicieux à retenir pour démarrer la résolution, puis ceux qu'il est nécessaire d'ajouter au cours de la résolution (Fig. 6).

$$
J_{Q}(\mathrm{~d} \boldsymbol{a})=\frac{1}{2}\|\boldsymbol{P} \boldsymbol{R}\|_{Q}^{2}
$$

La recherche du minimum de la fonction coût (21) passe par l'expression de sa dérivée (22), dans la direction da, pour tous vecteurs $\boldsymbol{\delta} \mathrm{d} \boldsymbol{a}$.

$$
\left\langle J_{Q}^{\prime}(\mathrm{d} \boldsymbol{a}), \boldsymbol{\delta} \mathrm{d} \boldsymbol{a}\right\rangle=\boldsymbol{R}^{\mathrm{T}} \boldsymbol{P} \boldsymbol{Q P} \boldsymbol{K}_{\mathrm{T}} \boldsymbol{\Phi}
$$

L'annulation de la dérivée (22) mène à la forme itérative (23) suivante.

$$
\left[\boldsymbol{\Phi}^{\mathrm{T}} \boldsymbol{K}_{\mathrm{T}}^{\mathrm{T}} \boldsymbol{P} \boldsymbol{Q} \boldsymbol{K}_{\mathrm{T}} \boldsymbol{\Phi}\right] \mathrm{d} \boldsymbol{a}=-\boldsymbol{\Phi}^{\mathrm{T}} \boldsymbol{K}_{\mathrm{T}}^{\mathrm{T}} \boldsymbol{P} \boldsymbol{Q} \boldsymbol{r}
$$

La matrice de rigidité tangente hyper réduite $\hat{\boldsymbol{K}}_{\mathrm{T}}$ est ainsi définie par l'équation (24), et le résidu hyper réduit $\hat{\boldsymbol{r}}$ par l'équation (25).

$$
\begin{gathered}
\hat{\boldsymbol{K}}_{\mathrm{T}}=\boldsymbol{\Phi}^{\mathrm{T}} \boldsymbol{K}_{\mathrm{T}}^{\mathrm{T}} \boldsymbol{P} \boldsymbol{Q} \boldsymbol{K}_{\mathrm{T}} \boldsymbol{\Phi} \\
\hat{\boldsymbol{r}}=-\boldsymbol{\Phi}^{\mathrm{T}} \boldsymbol{K}_{\mathrm{T}}^{\mathrm{T}} \boldsymbol{P} \boldsymbol{Q} \boldsymbol{r}
\end{gathered}
$$

Le choix de la norme $Q=\boldsymbol{I}$ mène à la forme itérative (26) suivante, pour une formulation en minimisation du résidu.

$$
\left[\boldsymbol{\Phi}^{\mathrm{T}} \boldsymbol{K}_{\mathrm{T}}^{\mathrm{T}} \boldsymbol{P} \boldsymbol{K}_{\mathrm{T}} \boldsymbol{\Phi}\right] \mathrm{d} \boldsymbol{a}=-\boldsymbol{\Phi}^{\mathrm{T}} \boldsymbol{K}_{\mathrm{T}}^{\mathrm{T}} \boldsymbol{P} \boldsymbol{r}
$$

Quant à la formulation de Galerkin, $Q=\boldsymbol{K}_{\mathrm{T}}^{-1}$, elle correspond à une matrice de rigidité tangente hyper réduite $\hat{\boldsymbol{K}}_{\mathrm{T}}=\boldsymbol{\Phi}^{\mathrm{T}} \boldsymbol{P} \boldsymbol{K}_{\mathrm{T}} \boldsymbol{\Phi}$.

L'algorithme de résolution, précédemment présenté (Fig. 1), est utilisé également ici, avec la matrice de rigidité tangente hyper réduite $\hat{\boldsymbol{K}}_{\mathrm{T}}$ et le résidu hyper réduit $\hat{\boldsymbol{r}}$. L'intérêt est ici de former et de réduire une matrice de rigidité tangente sur un nombre de degrés de liberté très réduit dans la boucle itérative Newton-Raphson. 

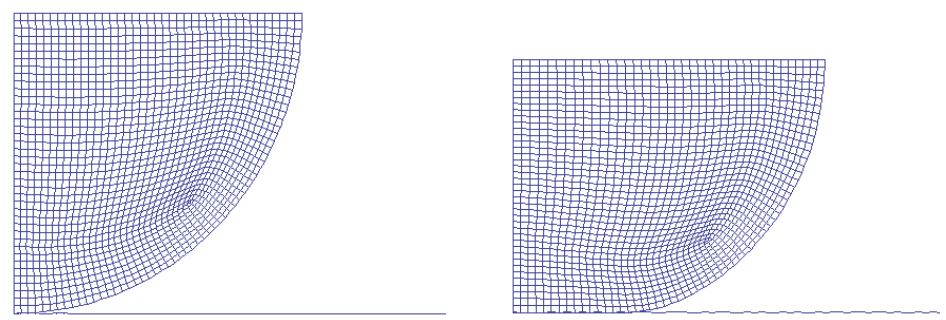

Fig. 2. Modèle élément-fini initial et déformée d'un quart du cylindre en caoutchouc.

\section{Application}

Nous allons maintenant appliquer les deux variantes proposées de méthode adaptative, avec utilisation de la technique POD pour gérer le contenu et la dimension de la base modale.

L'application présentée est un cylindre en caoutchouc écrasé sur un plan rigide. Le modèle est donc à nonlinéarité géométrique, matérielle et de contact. Un modèle néo-hookéen est utilisé pour le matériau, et la gestion du contact mécanique sans frottement est faite à l'aide d'un algorithme de Lagrangien augmenté.

Une étude 2D en déformation plane est proposée, avec un maillage à $n=3200$ degrés de liberté en éléments finis de type Q4 (Fig. 2) d'un quart du cylindre. L'écrasement du cylindre correspondant à $20 \%$ du rayon est constitué de 70 cas de charge successifs.

L'ensemble est modélisé sous le logiciel FEAP [16] pour mettre en place les données et construire la matrice de rigidité tangente et le résidu. La résolution (Fig. 1) est pour sa part réalisée à l'aide du logiciel MATLAB, les deux codes étant interfacés à l'aide de l'utilitaire FEAPMEX [17].

\subsection{Méthode adaptative avec POD}

L'algorithme de la figure 1 est ici appliqué au modèle du cylindre en caoutchouc, avec un base modale adaptative et régulée avec la technique POD. Nous allons comparer les formes itératives (7) et (8) correspondant respectivement à une formulation de Galerkin et à une formulation en minimisation du résidu.

Tout d'abord, nous observons le temps CPU (Central Processing Unit) nécessaire à la réalisation de la campagne de calculs pour les deux formulations envisagées (Fig. 3).

L'allure des deux courbes en temps CPU en fonction des cas de charge est sensiblement identique. Le gain en temps est de l'ordre de $11 \%$ pour la formulation de Galerkin comparée à la formulation par minimisation du résidu.

Puis, nous nous intéressons à la base modale, nous observons avec la figure 4 l'évolution de sa dimension en fonction des différents enrichissements et des différentes

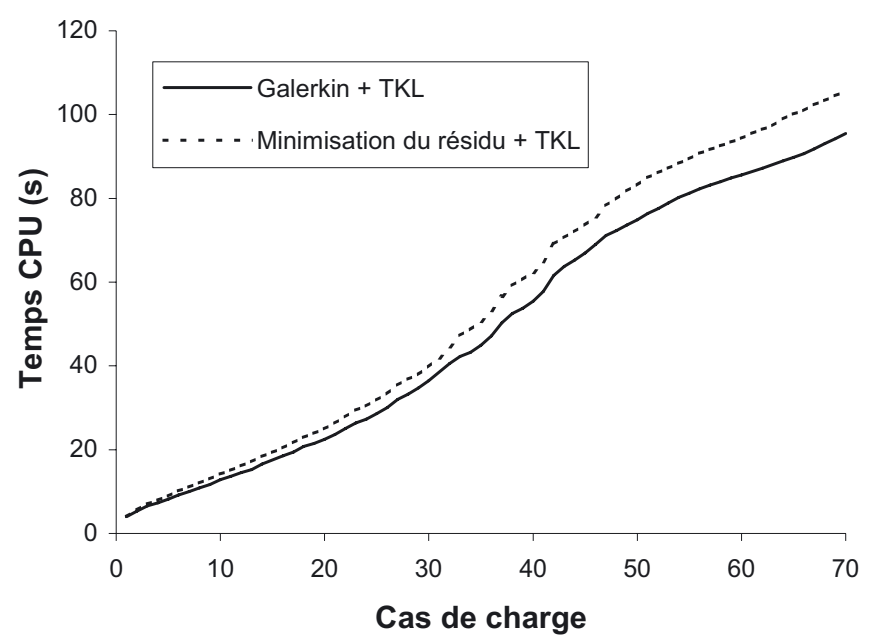

Fig. 3. Temps CPU (méthode adaptative avec POD) pour les deux formulations.

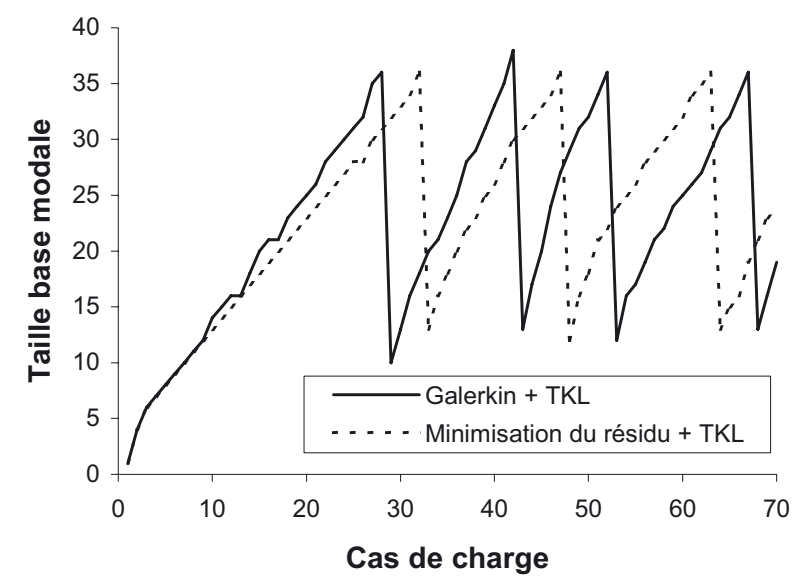

Fig. 4. Taille de la base modale (méthode adaptative avec POD) pour les deux formulations.

réductions POD pendant la réalisation de la campagne de calculs.

Des tests préliminaires ont permis de fixer la valeur limite $m_{\text {limit }}=35$ modes, afin de contrôler la taille de la base modale et donc minimiser le coût de la matrice de rigidité tangente réduite des équations (7) et (8).

Pour les deux formulations, Galerkin et minimisation du résidu, l'allure de l'évolution de la dimension de la base 
Tableau 1. Paramètres (méthode adaptative avec POD) pour les deux formulations.

\begin{tabular}{|l|c|c|}
\hline & Galerkin & Minimisation du résidu \\
\hline Nombre d'itérations Newton-Raphson & 1040 & 1020 \\
Nombre d'enrichissements de la base modale & 135 & 108 \\
\hline
\end{tabular}

Tableau 2. Paramètres (méthode adaptative d'hyper réduction avec POD) pour les deux formulations.

\begin{tabular}{|c|c|c|}
\hline & Galerkin & Minimisation du résidu \\
\hline Nombre d'itérations Newton-Raphson & 1129 & 1055 \\
\hline ddl retenus à chaque enrichissements de la base & 25 & 1 \\
\hline ddl retenus cumulés en fin de campagne & 774 & 304 \\
\hline Nombre d'enrichissements de la base modale & 173 & 138 \\
\hline
\end{tabular}

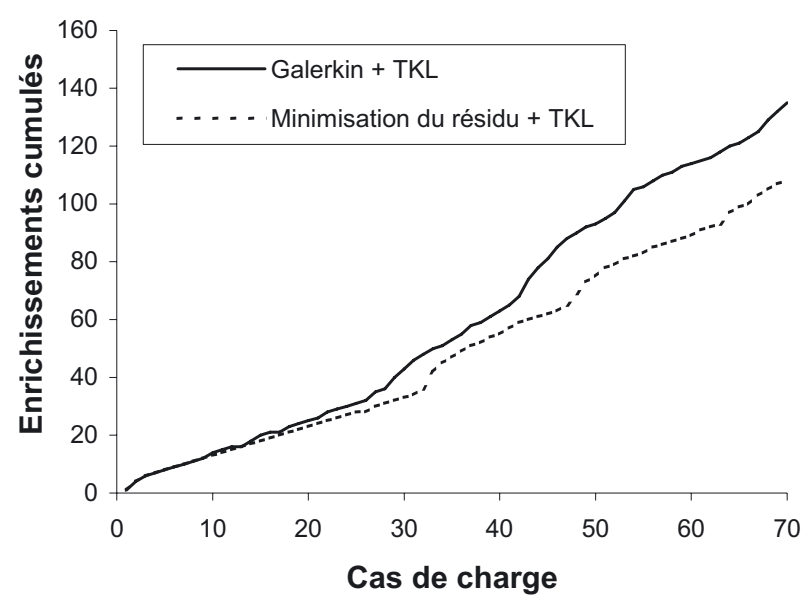

Fig. 5. Enrichissements cumulés de la base modale (méthode adaptative avec POD) pour les deux formulations.

modale est en dents de scie, induite par chaque réduction POD. Nous observons en effet que la technique POD permet de réduire le nombre $m$ de modes de 35 à un nombre $m^{\prime}$ souvent proche de 10 , pour le critère (17) fixé à $10^{-8}$.

Avec la formulation de Galerkin, il y a davantage d'enrichissements, essentiellement au cours de la première moitié de la campagne de calculs. La première réduction POD se fait plus tôt, et à l'issue des cas de charge, quatre réductions $\mathrm{POD}$ ont été réalisées pour la formulation de Galerkin contre trois pour la formulation en minimisation du résidu.

Nous pouvons observer en effet cette différence du nombre d'enrichissements de la base modale sur la figure 5 .

Suite à chaque réduction POD, davantage d'enrichissements sont effectués, la base modale fortement réduite a besoin de quelques modes supplémentaires. Au terme de la campagne de calculs, 135 enrichissements (Tab. 1) et 4 POD ont été réalisés pour la formulation de Galerkin, contre 108 et 3 POD pour la formulation en minimisation du résidu.

Malgré un coût d'enrichissement de la base plus important pour la formulation de Galerkin, cette dernière est légèrement plus intéressante en temps CPU (Fig. 3). Ceci provient du coût de la formation de la matrice tangente réduite vue dans l'expression itérative (7).
Pour chaque formulation, le coût de cette méthode adaptative avec POD (Algorithme 1) est réparti entre le coût des itérations de Newton-Raphson et le coût de l'enrichissement de la base modale, soit le tableau 1.

Ces données de la campagne de calculs réalisée sont à comparer à la résolution Newton-Raphson sur le déplacement $\boldsymbol{u}$, qui nécessite 1354 itérations pour une matrice de rigidité tangente à $n$ degrés de liberté. Les deux formulations requièrent ici moins d'itérations Newton-Raphson, sur le coefficient $\boldsymbol{a}$, soit un écart de l'ordre de $20 \%$, pour une matrice de rigidité tangente réduite à $m$ degrés de liberté (7), (8). Quant à la procédure d'enrichissement de la base modale, les formulations de Galerkin et de minimisation du résidu induisent respectivement 135 et 108 enrichissements, le coût est ici une itération de Newton-Raphson sur le déplacement $\boldsymbol{u}$. Le coût global en temps CPU est ainsi divisé par 2 avec la méthode adaptative pour les deux formulations.

Le modèle complet du cylindre comporte 3200 degrés de liberté. Nous venons de voir que la méthode adaptative avec POD permet de décrire le comportement de ce modèle avec 10 modes environ, une fois la POD appliquée.

\subsection{Méthode adaptative d'hyper réduction avec POD}

Nous allons maintenant réaliser les mêmes essais numériques sur le cylindre en caoutchouc avec la méthode adaptative d'hyper réduction, associée avec la technique POD.

D'une part, les degrés de liberté initialement choisis pour démarrer la campagne de calculs sont uniquement ceux concernés par les conditions aux limites et le chargement (Fig. 6, cas de charge $k=1$ )

D'autre part, pour chaque formulation, Galerkin et minimisation du résidu, différents tests sont effectués pour déterminer le nombre de degrés de liberté (ddl) retenus à chaque enrichissement de la base modale afin d'assurer la convergence. Chaque nouveau mode rajouté à la base est calculé en fonction des ddl retenus. Ceux-ci sont décrits dans le tableau 2 suivant. Les ddl choisis sont ceux pour lesquels le déplacement est maximal.

Ce tableau, ainsi que la figure 6, nous montrent que la convergence de la formulation de Galerkin nécessite davantage de ddl pour l'ajout d'un nouveau mode dans la base modale. La formulation en minimisation du résidu, 

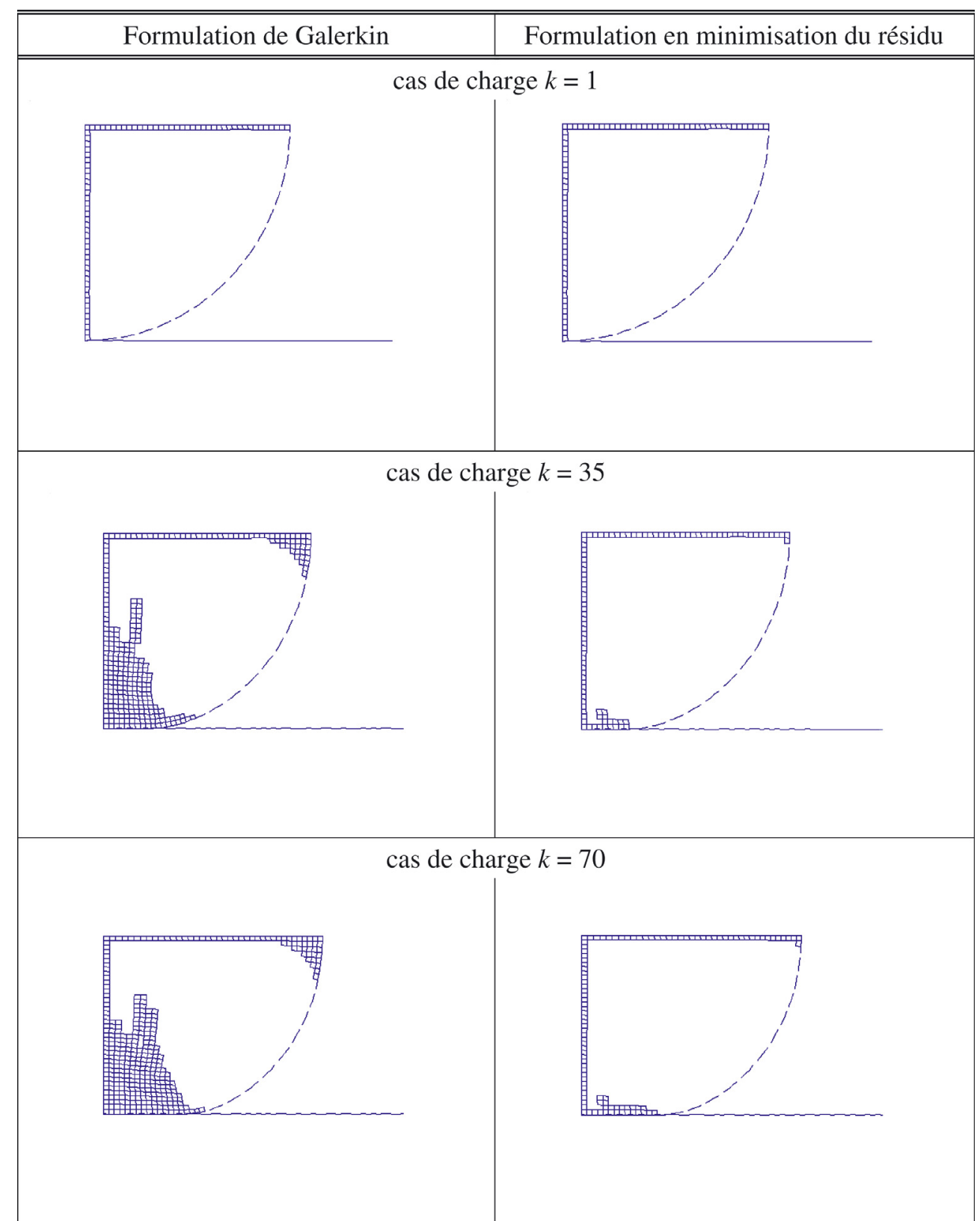

Fig. 6. Éléments-finis associés aux ddl retenus pour les deux formulations au cours de la campagne de calculs constituée de 70 cas de charge.

quant à elle, se contente d'ajouter un seul ddl. Par conséquent, le coût itératif de l'équation (24) est plus intéressant pour la formulation en minimisation du résidu, avec un nombre d'itérations de Newton-Raphson qui n'est pas plus important (Tab. 2). Nous observons que la dimension de la matrice de rigidité tangente hyper réduite (25) est très inférieure aux 3200 ddl du modèle complet (Fig. 2). A l'issue du chargement complet (cas de charge $k=70$ ), le modèle hyper réduit comporte $774 \mathrm{ddl}$ pour la formulation de Galerkin, et 304 ddl pour la formulation en minimisation du résidu.

La figure 6 nous renseigne également sur la localisation des degrés de liberté utiles à la résolution du système hyper réduit. Pour chaque formulation, en plus des ddl concernés par les conditions de symétrie et le chargement, les ddls ajoutés à chaque enrichissement de la base modale sont situés essentiellement près de la zone de contact, là où les non-linéarités sont concentrées.

Le coût CPU de la méthode adaptative d'hyper réduction est présenté, pour chaque formulation, sur la figure 7. Nous avons déjà précisé que le coût itératif Newton-Raphson était moindre pour la formulation en minimisation du résidu; ceci est également observé sur le coût global CPU où l'écart est de $28 \%$.

Nous allons maintenant regarder le coût de la base modale et de son enrichissement qui est à considérer dans le coût global de la méthode adaptative d'hyper réduction. 


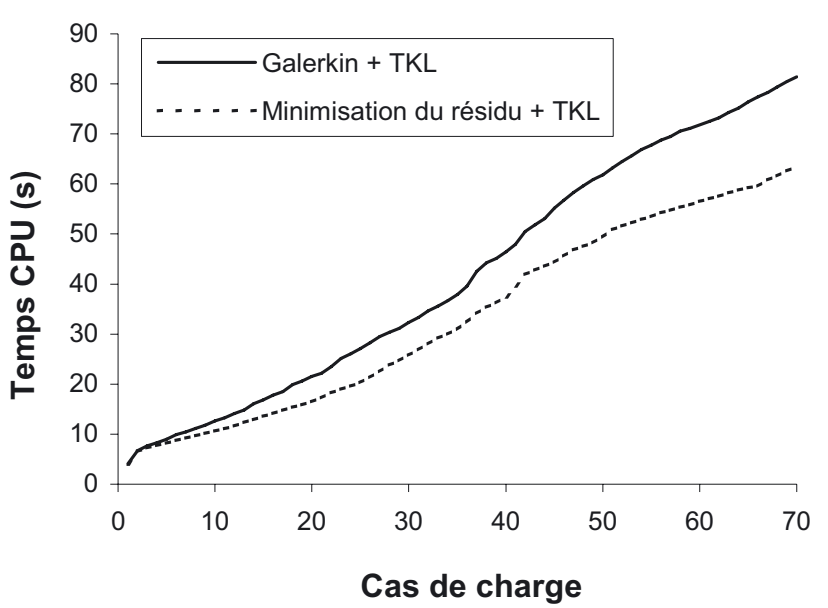

Fig. 7. Temps CPU (méthode adaptative d'hyper réduction avec POD) pour les deux formulations.

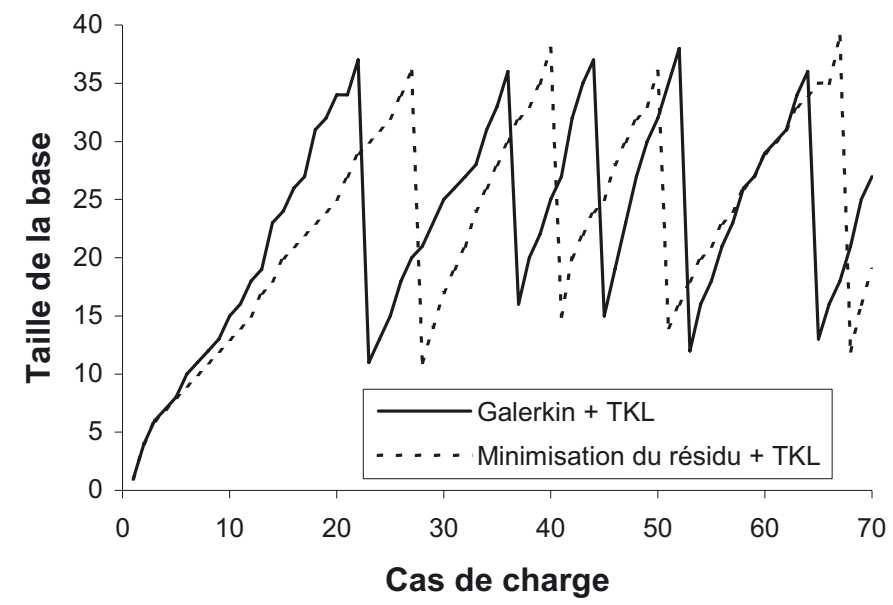

Fig. 8. Taille de la base modale (méthode adaptative d'hyper réduction avec $\mathrm{POD}$ ).

L'allure de la dimension de la base modale, ainsi que le nombre de réductions POD, sont similaires (Fig. 8) à ce qui avait été montré à la section précédente pour la méthode adaptative sans hyper réduction.

Le cumul du nombre d'enrichissements (Fig. 9) confirme que la formulation en minimisation du résidu est la moins coûteuse (gain de $22 \%$ ).

Le coût de la méthode adaptative d'hyper réduction avec POD est réparti, comme pour la méthode adaptative sans hyper réduction, entre le coût des itérations de Newton-Raphson sur le coefficient $\boldsymbol{a}$ et le coût de l'enrichissement de la base modale (Tab. 2).

Le modèle complet du cylindre (Fig. 2) est constitué de 3200 degrés de liberté. La méthode adaptative d'hyper réduction avec $\mathrm{POD}$, pour une formulation en minimisation du résidu, n'utilise au final que 304 degrés de liberté (Tab. 2 et Fig. 6) pour résoudre le problème du cylindre écrasé hyperélastique. Son comportement est décrit avec une base modale de 10 modes au minimum, et 35 au maximum.

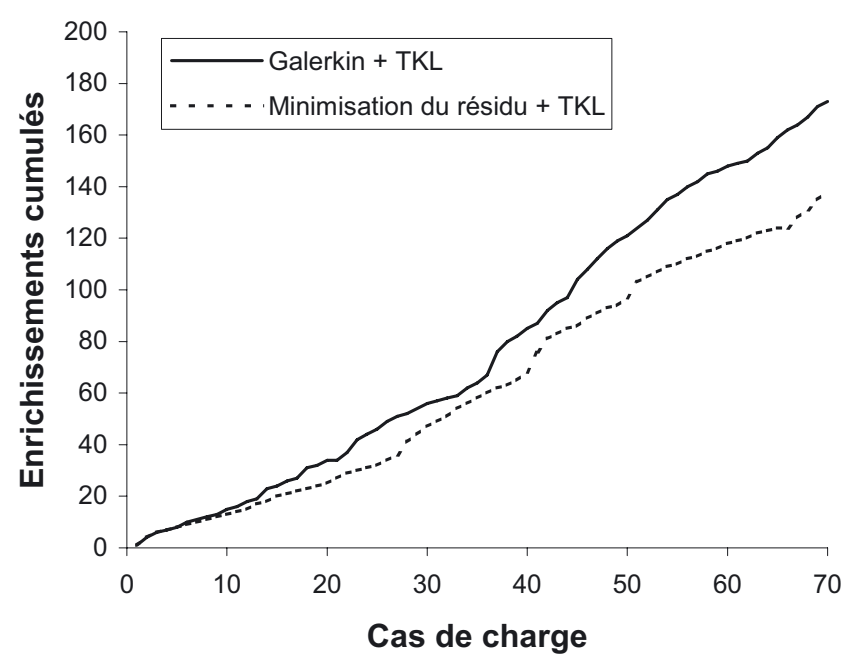

Fig. 9. Enrichissements cumulés (méthode adaptative d'hyper réduction avec $\mathrm{POD}$ ) pour les deux formulations.

\subsection{Discussion}

Nous allons faire ici le bilan du coût numérique et de la réduction de données pour les méthodes de réduction de modèle présentées. Nous venons de voir que la méthode adaptative avec hyper réduction, pour la formulation en minimisation du résidu, apportait les résultats les plus satisfaisants. En effet la réduction est de l'ordre de $40 \%$ par rapport à la méthode adaptative sans hyper réduction.

Et maintenant, comparée à une méthode classique d'itérations Newton-Raphson sur le déplacement $\boldsymbol{u}$, le gain pour la méthode adaptative d'hyper réduction est de l'ordre de 3,4 en temps CPU. La réduction des données est également très intéressante, en vue d'une phase d'immersion temps réel. Puisque, la méthode adaptative d'hyper réduction (formulation en minimisation du résidu) démarre la campagne de calculs avec 264 degrés de liberté, localisés aux conditions de symétrie et sur la zone de chargement, et termine l'écrasement complet du cylindre avec 304 degrés de liberté, soit moins de $10 \%$ des ddls du modèle complet $(n=3200)$. Les ddls ajoutés au cours de résolution sont pris en compte dans la formation de la matrice tangente hyper réduite de l'équation (27) et sont utilisés également pour l'enrichissement de la base modale. Le nombre $m$ de modes de celle-ci varie (Fig. 8) entre 10 et 35 , soit un très faible nombre de modes pour décrire un champ de déplacement à 3200 degrés de liberté.

\section{Conclusion}

L'objectif de ce papier est d'étudier la faisabilité de construction d'une surface de réponse réduite en vue d'être utilisée dans un simulateur temps réel de pièce déformable avec contact mécanique. Pour ce faire, des méthodes de réduction de modèle ont été utilisées, soient trois niveaux de réduction. Une méthode a priori avec base modale adaptative décrivant le champ de 
déplacement recherché, la décomposition orthogonale aux valeurs propres $\mathrm{POD}$ pour réguler cette base, et une variante de la précédente méthode a priori avec hyper réduction. Ces approches adaptatives ont été testées, pour une formulation de Galerkin et pour une formulation en minimisation du résidu, sur un problème d'écrasement de cylindre en caoutchouc sur un plan rigide. La résolution par une méthode adaptative de ce problème à non linéarités géométrique, matérielle et de conditions aux limites, a fourni des gains intéressants en temps CPU, et surtout en réduction de données par rapport à une résolution classique avec un schéma de Newton-Raphson sur le champ de déplacement. Plus particulièrement, la méthode adaptative d'hyper réduction, pour une formulation en minimisation du résidu, a donné les meilleurs résultats.

Un modèle non linéaire est donc ici correctement décrit par une surface de réponse réduite, pour laquelle les données seraient exploitables dans une simulation temps réel, dans le but de tester ce modèle d'une manière interactive.

\section{Références}

[1] http://www .haption.com

[2] D. James, D.K. Pai, ArtDefo, Accurate Real Time Deformable Objects, Computer Graphics ACM SIGGRAPH 99 Conference Proceedings, 1999, pp. 65-72

[3] S. Cotin, H. Delingette, N. Ayache, A Hybrid Elastic Model allowing Real-Time Cutting, Deformations and Force-Feedback for Surgery Training and Simulation, Vis. Comput. 16 (2000) 437-452

[4] C. Duriez, C. Andriot, A. Kheddar, Signorini's contact model for deformable objects in haptic simulations, IEEE/RSJ International Conference on Intelligent Robots and Systems, Sendai, Japon, 2004

[5] N. Talbi, P. Joli, Z.-Q. Feng, A. Kheddar, Real time simulation of interaction between deformable objects with haptic feedback for solving friction multiple contacts, GRAPP 2008-Third International Conference on Computer Graphics Theory and Applications, Funchal, Madeira, Portugal, 2008

[6] S. Niroomandi, I. Alfaro, E. Cueto, F. Chinesta, Realtime deformable models of non-linear tissues by model reduction techniques, Comp. Meth. Progr. Biomedicine 91 (2008) 223-231

[7] M.M. Loève, Probability Theory, Van Nostrand, New Jersey, 1955

[8] J.L. Dulong, F. Druesne, P. Villon, A model reduction approach for real-time part deformation with nonlinear mechanical behavior, Int. J. Interact. Des. Manuf. 1 (2007) 229-238

[9] D. Ryckelynck, A priori hyperreduction method: an adaptive approach, J. Computat. Phys. 202 (2005) 346-366

[10] M. Fortin, R. Glowinski, Augmented lagrangian methods : Application to the numerical solution of boundary value problems, North-Holland, Amsterdam, 1983

[11] B. Lefevre, F. Druesne, J.L. Dulong, P. Villon, Simulation of a mechanical assembly using model reduction, The 8th World Congress on Computational Mechanics WCCM8, Venise, 2008

[12] J. Lumley, The structure of inhomogeneous turbulent flows, Atmospheric Turbulence and Radio Wave Propagation, in A.M. Yaglom and Tararsky (ed.), 1967, pp. 166-178

[13] P. Krysl, S. Lall, J.E. Marsden, Dimensional Model Reduction in Non-linear Finite Element Dynamics of Solids and Structures, Int. J. Num. Meth. Eng. 51 (2000) 479-504

[14] N. Verdon, C. Allery, A. Hamdouni, D. Ryckelynck, Reduced-Order Modelling for solving linear and nonlinear equations, Commun. Numer. Meth. Eng. (2010)

[15] D. Ryckelynck, Missoum Benzianze D., Multi-level a priori hyper-reduction of mechanical models involving internal variables, Comput. Methods Appl. Mech. Eng. 199 (2010) 1134-1142

[16] http://www.ce.berkeley.edu/feap

[17] http://www.cims.nyu.edu/ dbindel/feapmex/ feapmex/doc/feapmex.html 